\title{
Investigating the effect of sterilisation methods on the physical properties and cytocompatibility of methyl cellulose used in combination with alginate for 3D-bioplotting of chondrocytes
}

\author{
Ella Hodder ${ }^{1,2 * \#}$, Sarah Duin ${ }^{3 *}$, David Kilian ${ }^{3}$, Tilman Ahlfeld ${ }^{3}$, Julia Seidel ${ }^{3,4}$, Carsten Nachtigall ${ }^{4}$, Peter \\ Bush $^{2}$, Derek Covill ${ }^{1}$, Michael Gelinsky ${ }^{3}$ and Anja Lode ${ }^{3 \#}$ \\ ${ }^{1}$ School of Computing, Engineering and Mathematics, University of Brighton, United Kingdom \\ ${ }^{2}$ School of Pharmacy and Biomolecular Science, University of Brighton, United Kingdom \\ ${ }^{3}$ Centre for Translational Bone, Joint and Soft Tissue Research, Faculty of Medicine Carl Gustav Carus, \\ Technische Universität Dresden, Germany \\ ${ }^{4}$ Institute of Natural Materials Technology, Technische Universität Dresden, Germany \\ *Both authors contributed equally to this work
}

"Corresponding authors: E.Hodder@brighton.ac.uk_anja.lode@tu-dresden.de

\begin{abstract}
For both the incorporation of cells and future therapeutic applications the sterility of a biomaterial must be ensured. However, common sterilisation techniques are intense and often negatively impact on material physicochemical attributes, which can affect its suitability for tissue engineering and 3D printing. In the present study four sterilisation methods, autoclave, supercritical $\mathrm{CO}_{2}\left(\mathrm{scCO}_{2}\right)$ treatment, UV- and gamma $(\gamma)$ irradiation were evaluated regarding their impact on material properties and cellular responses. The investigations were performed on methyl cellulose (MC) as a component of an alginate/methyl cellulose (alg/MC) bioink, used for bioprinting embedded bovine primary chondrocytes (BPCs). In contrast to the autoclave, $\mathrm{scCO}_{2}$ and $\mathrm{UV}$-treatments, the $\gamma$-irradiated $\mathrm{MC}$ resulted in a strong reduction in alg/MC viscosity and stability after extrusion which made this method unsuitable for precise bioprinting. Gel permeation chromatography analysis revealed a significant reduction in $\mathrm{MC}$ molecular mass only after $\gamma$-irradiation, which influenced $\mathrm{MC}$ chain mobility in the $\mathrm{Ca}^{2+}$ crosslinked alginate network as well as gel composition and microstructure. With regard to cell survival and proteoglycan matrix production, the results determined UV-irradiation and autoclaving as the best candidates for sterilisation. The $\mathrm{scCO}_{2}$-treatment of $\mathrm{MC}$ resulted in an unfavourable cell response indicating that this method needs careful optimisation prior to application for cell encapsulation. As proven by consistent FT-IR spectra, chemical alterations could be excluded as a cause for the differences seen between MC-treatments on alg/MC behaviour. This investigation provides knowledge for the development of a clinically appropriate 3D-printing-based fabrication process to produce bioengineered tissue for cartilage regeneration.
\end{abstract}


Key words: autoclaving, UV-irradiation, gamma irradiation, supercritical $\mathrm{CO}_{2}$, bioprinting, methyl cellulose, hydrogels, bioink, biomaterial, additive manufacturing

\section{Introduction}

Additive manufacturing (AM) is an automated production process that encompasses a series of computationally directed, layer-by-layer, printing techniques to generate highlycontrolled customisable 3D objects. Due to its high throughput efficiency and quality control features the application of AM for tissue engineering is an area of increasing interest, capable of using data derived from medical imaging to create versatile and precise, clinically relevant scaffolds [1-2]. Three-dimensional extrusion-based bioplotting is an AM method with the unique ability to produce volumetric scaffolds with encapsulated cells; this has been achieved using various biomaterial and cell combinations. In particular, hydrogels offer a popular choice for this purpose due to their high water contents, biocompatibility, and rheological properties [3-4].

Alginate is a naturally occurring anionic polymer and represents one of the most extensively investigated hydrogels used for many biomedical applications [5]. Due to its simple crosslinking by multivalent cations and its suitability for cell encapsulation [6] the transition of alginate for extrusion-based 3D bioprinting has been commonly pursued [7-8]. However, this has not been without challenge [9]. Materials suitable for extrusion must not only ensure continuous fluid-flow, but also maintain structural integrity upon deposition [3]. Hence, viscous pastes are highly suitable whereas alginate solutions at low concentrations have a poor applicability for plotting of 3D scaffolds due to its fluid like state [9-10]. However, a simple increase of its concentration to enhance the viscosity is restricted by a related decrease in cell survival after crosslinking. Alternative methods to improve hydrogel print capabilities without an increase in alginate network density include the use of complimentary material combinations, to utilise different gelation properties such as, alginate-gelatine [11], alginatefibrin [12] and alginate-methyl cellulose [10].

The use of methyl cellulose (MC) as a thickening additive for alginate extrusion-based 3D printing is a relatively new approach, with the first formulation introduced by Schütz et al [10] (first available online in 2015). It has since demonstrated a high potential for the bioplotting of different cell types [13-16] and its relevance for use in 3D printing was recently highlighted [17]. MC is obtained from the bacterium Gluconacetobacter xylinus and possesses high crystallinity, purity, and a large capacity for water absorption with excellent mechanical properties including shear thinning. MC is an easily mass produced, non-toxic and non-allergen substance with a current commercial importance across a range of industries, such as food and cosmetics [18]. It is also commonly used in stem cell culture techniques [19].

Prior to the incorporation of cells, the sterility of a biomaterial must be ensured. Sterilisation refers to the complete destruction of life and biological agents including fungi, bacteria and viruses. present within a targeted region [20]. It is commonly achieved via the application of heat, chemicals, irradiation, high pressure or filtration through membranes with $0.22 \mu \mathrm{m}$ pore sizes [21]. However, by design common sterilisation techniques are intense, and can cause change to a material's chemical and physical properties [20-21]. These alterations 
can affect its performance and feasibility for extrusion and tissue engineering. It is therefore of high importance to select the most appropriate sterilisation procedure, specific to the material used and its intended application.

Autoclaving is one of the most widely used health care sterilisation techniques due to its speed, efficacy and low cost [22]. The method uses pressurised steam and high temperature water for the thermal destruction of microbial life. However, high temperatures have been reported to adversely affect sensitive biomaterials such as polysaccharides [21,23].

A low temperature alternative is the application of supercritical carbon dioxide $\left(\mathrm{scCO}_{2}\right)$ in combination with low amounts of chemical additives. The basis of this sterilisation method is through the extreme pressurisation of $\mathrm{CO}_{2}$ to nearly 10 times atmospheric pressure, above the critical point when the gas forms a solvent [24]. Although the precise mechanism by which the $\mathrm{scCO}_{2}$ method sterilises samples is not yet fully investigated, it is proposed to be the result of intracellular reactions upon gas diffusion into the cell which cause the formation of carbonic acid, leading to acidification and this, along with chemical oxidation can kill bacteria [25-26]. The use of $\mathrm{scCO}_{2}$ has been shown to achieve sterilisation without any notable negative physical or structural effects and has been suggested as a promising treatment for use with sensitive biomaterials [27]. In particular, the application of $\mathrm{scC}_{2}$ on $\mathrm{MC}$ was shown to be less compromising on mechanical and rheological properties when directly compared to ethylene oxide exposure, another low temperature alternative [26].

Gamma $(\gamma)$ irradiation is the most common sterilisation technique for orthopaedic implants [28], as well as various other fields including food preservation and pharmaceutical production [29], with between 25 and $40 \mathrm{kGy}$ doses of radiation known to ensure sterility. Energy from photons of $\gamma$ radiation is transferred to the electrons in the exposed material [30]. The highly excited electrons then yield high doses of free radicals which disrupt the DNA double helix of any microorganism present. When the DNA is irreparably destroyed the cells are no longer able to function. However, $\gamma$-irradiation has been linked to crosslinking and chain scission in some polymers [31-33].

Ultra-violet (UV) light is employed in a comparable way to $\gamma$-irradiation but uses lower energy photons; with wavelengths between 290 and $200 \mathrm{~nm}$ considered optimal for disinfection purposes [34]. The UV rays work by damaging DNA through initiating a reaction between two molecules of thymine within the nucleic acid. Dependent on the duration and proximity of the UV source, the DNA is irreparably destroyed, and the cells become inert [35]. Although UVtreatment is not considered a fully effective method for the terminal stage sterilisation of all biological life [31], it is a common method of choice for material sterilisation in research laboratories [36-39].

Acknowledging the high impact of sterilisation on biopolymer properties with respect to fabrication and scaffold characteristics, the intention of our present study was to systematically investigate the effect of four sterilisation methods on material properties relevant for bioplotting and cartilage tissue engineering. In addition to the easily accessible and commonly employed UV and autoclave treatments, we tested the well-established $\gamma$-irradiation and the less utilised scCO2 sterilisation as a low temperature and irradiation-free method. We focused on the effect of sterilisation on methyl cellulose and for its role as a universal thickening agent for hydrogel plotting, to enable cell compatible and improved printing properties for the fabrication of volumetric, open-porous constructs. Hence, the sterilisation treatments were 
performed on powdered MC, as part of the alg/MC blend previously developed by Schütz et al [10]. The sterilisation of the alginate component, by autoclaving, was kept constant throughout, due to the low viscosity of $3 \%$ alginate solutions regardless of sterilisation method employed [40-41], and the already well established use of autoclave for alginate [40-44]. This allowed us to reliably attribute observed changes in the paste and/or scaffolds back to the effects of the MC treatment only, as the primary material conferring printability. By applying Fouriertransform infrared spectroscopy (FTIR) and gel permeation chromatography (GPC) the chemical composition and molecular mass of the treated MC types were evaluated. The resulting alg/MC pastes were characterised with respect to viscosity and suitability for extrusion and the plotted scaffolds were analysed based on microstructure, composition, and mechanical properties. Finally, bovine primary chondrocytes (BPCs) were embedded and cell viability as well as extracellular matrix deposition within the scaffolds were evaluated.

\section{Materials and methods}

\section{Materials}

Dulbecco's modified eagle medium (DMEM), Hanks balanced salt solution (HBSS), penicillin/streptomycin \& phosphate buffered saline solution (PBS) from Thermofisher, USA; Foetal calf serum (FCS), gentamycin, MQuantTM Peracetic Acid Test kit, sodium citrate \& Trypan blue from Merck Millipore, USA; 5-chloromethylfluorescein diacetate (CMFDA) Green \& ethidium homodimer from Molecular probes, USA; acetic anhydride, alginic acid sodium salt, hydrogen peroxide $\left(\mathrm{H}_{2} \mathrm{O}_{2}\right)$, methyl cellulose \& safranin-O from Sigma-Aldrich, USA; calcium chloride $\left(\mathrm{CaCl}_{2}\right)$ \& chlorine-zinc-iodine solution from Roth, Germany; Mykoval from Hund Wetzlar, Germany; QuantiFluor dsDNA system from Promega, USA; formaldehyde solution from SAV LP GmbH, Germany; QUANTOFIX® Fluitest CA CPC assay from Analyticon Biotechnologies, Germany; Peroxidetest strips from Macherey-Nagel, Germany.

\section{Tissue processing and cell culture}

Primary bovine articular cartilage was isolated from bovine metacarpal phalangeal joints of skeletally mature animals ( $\sim 30$ months) as previously described by Hall et al [45]. Briefly, bovine lower limbs were obtained from a local abattoir and used within 4 hours of animal death. Full depth articular cartilage was removed under aseptic conditions and incubated for $\sim 20$ hours in the presence of collagenase (Type II; $2 \mathrm{mg} / \mathrm{ml}^{-1}$ ) in high glucose DMEM with $10 \mu \mathrm{g} / \mathrm{ml}$ gentamycin at $37^{\circ} \mathrm{C}$ and $5 \% \mathrm{CO}_{2}$. The digest was crudely filtered through a stainless-steel mesh followed by $70 \mu \mathrm{m}$ nylon sieve (BD Bioscience, USA). The collected cell suspension was washed by centrifugation ( 5 minutes at $400 \mathrm{xg}$ ) and counted by haemocytometer in the presence of trypan blue to determine cell viability. Cells were expanded for four days under normal cell culture conditions, seeded at $0.5 \times 10^{6}$ per T25 flask at $37^{\circ} \mathrm{C}$ and $5 \% \mathrm{CO}_{2}$ in culture medium consisting of DMEM with $10 \%$ FCS, $100 \mu \mathrm{g} / \mathrm{ml}$ penicillin and $100 \mu \mathrm{g} / \mathrm{ml}$ streptomycin. Viability of isolated cells typically varied between $80-90 \%$ following incubation. The isolated cells were then frozen after one passage (P1) at $-1{ }^{\circ} \mathrm{C} / \mathrm{min}$ and stored in liquid nitrogen for use within 2 months. The bovine primary chondrocytes (BPCs) were thawed and expanded in monolayer culture using previously stated cell culture conditions and growth medium. The 
culture medium was changed twice a week. Cells in passage 4 were used for bioplotting experiments.

\section{Sterilisation}

For each approach, a pre-weighed amount of methyl cellulose powder was transferred to Heat Seal Closure Pouches SteriBags ${ }^{\mathrm{TM}}$ (Fisher Scientific, USA). $\mathrm{ScCO}_{2}$ treatment was performed in a $750 \mathrm{ml}$ stainless steel autoclave (J\&W Scientific, Inc., USA). The MC powder was placed into the autoclave and an additive mixture of 0.25 vol\% water, 0.15 vol\% $\mathrm{H}_{2} \mathrm{O}_{2}$ and $0.5 \mathrm{vol} \%$ acetic anhydride was pipetted to the bottom of the vessel. The autoclave was then filled with $300 \mathrm{~g}$ liquid $\mathrm{CO}_{2}\left(18^{\circ} \mathrm{C}, 5.5 \mathrm{MPa}\right)$ and heated to transfer the liquid $\mathrm{CO}_{2}$ to the supercritical state $\left(\sim 38^{\circ} \mathrm{C}, \sim 8.5 \mathrm{MPa}\right)$ for 45 minutes, depressurisation was performed within 6.5 minutes. To check for chemical residues, $\mathrm{MC}$ was dissolved at a concentration of $2 \mathrm{~g} / \mathrm{L}$ in $\mathrm{ddH}_{2} \mathrm{O}$ after sterilisation with $\mathrm{scCO}_{2}$ and tested for $\mathrm{H}_{2} \mathrm{O}_{2}$ and peracetic acid with semiquantitative test strips. Untreated $\mathrm{MC}$ was tested as a negative control. For autoclaving, the MC powder was subjected to an autoclave cycle that consisted of high-pressure saturated steam at $121{ }^{\circ} \mathrm{C}$ and held for 20 minutes before cooling in a Systec D-23 table-top autoclave. For UVirradiation, the transparent MC bag was placed under a UVC lamp (wavelength $254 \mathrm{~nm}$, Fisher Scientific) at a distance of $5 \mathrm{~cm}$ for two hours (conditions selected based on previously employed studies shown to be within the range for effective sterilisation [46-48]). The powder was spread out and evenly distributed to ensure a thin layer across the inside surface and readjusted after an hour interval to allow for full UV light exposure. The process was performed within a laminar flow hood. Gamma irradiation was carried out using a Cobalt 60 source at minimal dose of $25 \mathrm{kGy}$ by BBF Sterilisation Service (Kernen, Germany). The sterilisation methods employed were previously validated using microbial cells by Bernhardt et al. [26], with the exception of UV a broadly implemented but currently non-approved (medium inactivation level) method for biomaterial sterilisation [22].

\section{Plotting material preparation}

The alg/MC hydrogel mixture was prepared as first described by Schütz et al [10]. In brief, a $3 \mathrm{wt} \%$ alginate solution was made by mixing alginic acid sodium salt in PBS and stirred overnight before sterilisation using autoclave $\left(121^{\circ} \mathrm{C}\right.$ for 20 minutes $)$ that has been shown to not alter the chemical structure of alginate (see supplementary Fig.S1). 9 wt\% sterilised MC was then added to the alginate solution. The mixture was stirred to obtain a homogeneous alg/MC paste and left to swell for 1.5 hours before the addition of BPCs. The cells were suspended in culture medium, with a ratio of cell suspension:plotting paste of 1:9, and a cell seeding density of $5 \times 10^{6}$ per gram of material. Cell free material was used for plotting of scaffolds for the evaluation of structure, composition and mechanical properties.

\section{D plotting of Alginate/methyl cellulose constructs}

The 3D plotting system used in this study was the BioScaffolder 3.1 from GeSiM mbH (Radeberg, Germany) operated under sterile conditions. The alg/MC material was dispensed through a dosing needle (Nordson GmbH, Germany) with an inner diameter of $610 \mu \mathrm{m}$ and using a plotting speed of $10 \mathrm{~mm} / \mathrm{s}$ and dosing pressure of 70-80 $\mathrm{kPa}$. For the extrusion of the $\gamma$-treated material a reduced print pressure of $65 \mathrm{kPa}$ bar and tip diameter of $450 \mu \mathrm{m}$ were required. The $3 \mathrm{D}$ constructs were built up layer-by-layer into 12-well plates by plotting into air. The structures were designed with the defined overall dimensions of $9.5 \mathrm{~mm}$ by $9.5 \mathrm{~mm}$, with a height of $1.4 \mathrm{~mm}$ per layer, comprised of 4 or 10 layers, 5 strands per layer, and an angle orientation change of $90^{\circ}$ between the layers. After print completion, the hydrogel scaffolds 
were crosslinked in $100 \mathrm{mM} \mathrm{CaCl}_{2}$ for 10 minutes. Both cell-laden and cell-free scaffolds were plotted under the same print conditions.

\section{Methyl cellulose characterisation}

\section{Fourier Transform Infrared Spectroscopy}

FT-IR (Fourier-transformed infrared) spectra were acquired from untreated and sterilised MC powders. The spectra were recorded with a 65 Fourier-transform infrared spectrometer (PerkinElmer), with an ATR crystal, in the spectral range of $400-4000 \mathrm{~cm}^{-1}$ at a resolution of $4 \mathrm{~cm}^{-1}$.

\section{Gel permeation chromatography}

The molecular mass of differently treated MC was determined by gel permeation chromatography (GPC). Samples with a concentration of $2 \mathrm{~g} / \mathrm{L}$ were filtered through a $0.45 \mu \mathrm{m}$ filter and measured at room temperature using a AZURA Assistant ASM 2.1 L (Knauer Wissenschaftliche Geräte GmbH, Germany) GPC system equipped with three columns (1x PSS Suprema $100 \AA$ \& 2x PSS Suprema $3000 \AA$ ), and a precolumn (PSS-Polymer -StandardService GmbH, Germany). The eluent consisted of $50 \mathrm{mM} \mathrm{NaNO}_{3}$ and $7.7 \mathrm{mM} \mathrm{NaN}_{3}$ in $\mathrm{dH}_{2} \mathrm{O}$. Calibration was carried out using Pullulan standards with defined molar masses ranging from $342 \mathrm{Da}$ to $2560 \mathrm{kDa}$ and a Smartline RI detector 2300. Molecular mass was calculated according to the following formulae:

$$
\begin{aligned}
& M_{w}=\frac{\sum N_{i} M_{i}^{2}}{\sum N_{i} M_{i}} \\
& M_{n}=\frac{\sum N_{i} M_{i}}{\sum N_{i}} \\
& P D I=\frac{M_{w}}{M_{n}}
\end{aligned}
$$

whereby $M_{w}$ is the molecular mass average of the weight, $M_{n}$ the number average molecular mass, and PDI the polydispersity index. $\mathrm{M}_{\mathrm{i}}$ denotes the molecular mass of a polymer chain and $\mathrm{N}_{\mathrm{i}}$ the number of chains within said molecular mass.

\section{alg/MC paste characterisation}

\section{Viscosity}

The viscosities of alg/MC pastes were determined at room temperature using a rheometer with plate-plate geometry (Rheotest RN 4, Germany), a plate diameter of $50 \mathrm{~mm}$, and a plateplate distance of $1 \mathrm{~mm}$. Shear thinning was tested for pastes with non-sterilised, autoclaved, UV - and $\gamma$-irradiated MC by constantly increasing rotational shear rate from 0 to $100 \mathrm{~s}^{-1}$ (increment $0.08 \mathrm{~s}^{-1}$ ). Before measurement the alg/MC material was left to swell at $4^{\circ} \mathrm{C}$ overnight, to avoid the effects of swelling on viscosity between measurements.

\section{Scaffold evaluation - structure and composition}

\section{Release of methyl cellulose}

The MC content within 3D plotted and $\mathrm{Ca}^{2+}$-crosslinked alg/MC scaffolds was observed via staining with a chlorine-zinc-iodine solution after 1, 7, 14 and 21 days. Images were taken using a stereo light microscope (Leica M205 C equipped with DFC295 camera, Germany). 
For quantitative analysis of MC release, alg/MC scaffolds were incubated in $2 \mathrm{ml}$ culture medium in cell culture conditions over the 21 -days investigation period. On defined time points ( $2 \mathrm{~h}$ and 1, 2, 4, 7, 11, 14, 19, 21 days) $1 \mathrm{ml}$ of supernatant was taken, and replaced by $1 \mathrm{ml}$ of fresh medium. Mykoval'TM (fluorophore formerly distributed as Calcofluor, Blankoflor or Uvitex 2B), a fluorescence marker primarily applied for cellulose and chitin detection in fungal cell walls [49-50] was used to determine soluble MC (see calibration data, supplementary Fig.S2). Method optimization was performed on scaffolds and pure MC solution samples at different concentrations $(3-750 \mu \mathrm{g} / \mathrm{ml})$, temperatures $\left(4^{\circ} \mathrm{C}, 26^{\circ} \mathrm{C}, 37^{\circ} \mathrm{C}\right)$ and media (DMEM, HBSS, $1 \mathrm{M}$ and $0.1 \mathrm{M} \mathrm{CaCl}_{2}$ ). Each calibration series used for the $\mathrm{MC}$ release calculation of the sterilisation methods was set up using equally treated MC. For measurements, $180 \mu \mathrm{l}$ of supernatant was mixed with $20 \mu 1$ Mykoval $^{\mathrm{TM}}$ solution in a black F-bottom polystyrene 96 well plate (Greiner Bio-One, Germany). The samples were incubated for 5 min protected from light and measured on a microplate reader (Infinite M200 Pro; Tecan, Switzerland), excitation and emission wavelengths used were 400 and $450 \mathrm{~nm}$ respectively. Regression analysis to obtain calibrated MC concentration via detected fluorescence signal was performed via non-linear regression (Model: one-site saturation) using GraphPad Prism 7.0 for Windows (GraphPAd Software, USA).

\section{Scanning electron microscopy (SEM)}

Scaffolds were washed twice with HEPES buffered saline (HBS) containing $\mathrm{Ca}^{2+}$ and $\mathrm{Mg}^{2+}$ and dehydrated using a gradation series of ethanol/distilled water solutions. Critical point drying was performed with a CPD 030 apparatus (BAL-TEC, Liechtenstein). Dried samples were coated with gold and imaged using a Philips XL 30/ESEM with field emission gun, operating in SEM mode.

\section{Calcium release}

The Fluitest CA CPC assay method was used to measure the $\mathrm{Ca}^{2+}$ concentration of the culture supernatants at progressive intervals during scaffold incubation. Cell-free scaffolds used for compression testing were incubated in $2 \mathrm{ml}$ cell culture medium, the medium was regularly changed (every 3 to 4 days) and $1 \mathrm{ml}$ of the supernatant was assessed against a known calcium standard according to manufacturer's instructions. Data was collected at an absorbance of $570 \mathrm{~nm}$.

\section{Compression testing}

Unconfined uni-axial compression experiments were performed using an Instron 5566 materials testing device equipped with a $100 \mathrm{~N}$ load cell (Instron Wolpert, Germany). Scaffolds plotted with 10 layers to reach a minimal height of $2 \mathrm{~mm}$ were investigated straight from culture in a wet state at room temperature with a compression load exerted at $5 \% / \mathrm{min}$ until failure. Compressive strength was determined by the maximum peak breaking point. Dimensional measurements of each scaffold were taken prior to compression using callipers and considered during device measurements.

\section{Alg/MC scaffold - cell compatibility}

\section{Cell survival}

Cell viability was determined via live/dead staining. After 1, 7, 14, and 21 days of incubation cell-laden scaffolds were incubated for 20 minutes in culture medium supplemented with $5 \mu \mathrm{M}$ CMFDA green and $1.4 \mu \mathrm{M}$ ethidium homodimer- 1 per $\mathrm{ml}$ at $37^{\circ} \mathrm{C}$ and $5 \% \mathrm{CO}_{2}$. Scaffolds were rinsed with HBSS, fixed in a $4 \%$ paraformaldehyde solution, and stored at $4{ }^{\circ} \mathrm{C}$ 
for a maximum of 2 weeks before imaging. Imaging was performed using a Zeiss Observer Z.1, wide field system with ApoTome (Zeiss, Germany) equipped with an hxp100 fluorescence lamp, GFP (EX 470/40, BS 495, EM 525/50) and RFP (EX 550/25, BS 570, EM 605/70) fluorescence filter cubes, and a Monochromatic Camera AxioCam 503 mono. Viable and dead cells were counted using the particle analyser of Image J V1.44p.

\section{DNA quantification}

To extract the DNA the alg/MC scaffolds were frozen at $-80^{\circ} \mathrm{C}$ on the days $1,7,14$, and 21 of culture, and dissolved in $3 \mathrm{ml}$ of $100 \mathrm{mM}$ sodium citrate. The cells were lysed at $60^{\circ} \mathrm{C}$ overnight followed by 10 minutes sonication. The lysate was stored at $-80^{\circ} \mathrm{C}$ until measurement of total DNA using the QuantiFluor dsDNA system as per the manufacturer's instructions.

\section{Glycosaminoglycan histology}

To localize sulphated glycosaminoglycans (sGAG) within the BPC-laden alg/MC constructs cultured for 21 days, histochemical analysis was performed using safranin O; cellfree scaffolds incubated for 21 days as well as BPC-laden constructs cultured for 1 day served as control. Scaffolds were fixed with $4 \%$ paraformaldehyde in HBSS (with $\mathrm{Ca}^{2+}$ and $\mathrm{Mg}^{2+}$ ), and then paraffin embedded using a tissue processor (LEICA TP 1050). Microtome sections of 10-micron thickness were mounted onto glass slides and proteoglycan analysed by staining with $0.1 \%$ safranin $\mathrm{O}$, Weigerts Iron Hematoxylin solution, and $0.02 \%$ counter stain fast green. Images were obtained by photomicroscopy using brightfield illumination, with the same light settings applied for each sample (Nikon, inverted microscope, ECLIPSE, TE2000).

Semi-quantitative results for the histological images were achieved using thresholding analysis on Image J V1.44p for area of stain coverage (8-bit threshold 54-140) and for stain intensity (hue threshold 0-29). Percent area coverage was calculated from the number of stained pixels divided by the images total picture number and multiplied by 100 . The relative stain intensity was based upon the grayscale of $0=$ black and $255=$ white. Data was normalised by dividing each grayscale value by the maximum (255) to invert the scale, for improved visual representation of stain intensity (higher value represents higher stain intensity). Two images per scaffold were analysed with three replicates per time point.

\section{Statistics}

For quantitative MC release studies, statistical analysis for triplicate samples were performed on GraphPad Prism via multiple t-tests with Bonferroni-Dunn correction, to compare single time points of the MC release curves between groups. For all other statistics the Mini-tab software package was used, data was initially tested for normality using the Anderson-Darling Test. The differences between multiple means where then compared via One-way ANOVA with post-hoc Tukey. The alpha level was set at 0.05.

\section{Results}

\section{MC characterisation}

To evaluate the potential impact of the sterilisation methods on the chemical composition of MC, the treated powders were analysed by Fourier-transform infrared spectroscopy in comparison to untreated MC. The FT-IR spectra revealed no significant difference between 
absorption bands for all MC variants tested, identified in Fig.1. The peak shapes and ratios were unchanged with no visual shifts or deformations (see supplementary Fig.S3), indicating that the $\mathrm{UV}$, autoclaved, $\mathrm{scCO}_{2}$, and $\gamma$ sterilisation processes did not modify the $\mathrm{MC}$ composition detectable by FT-IR.

The influence of the sterilisation treatments on the MC molecular weight was studied by gel permeation chromatography. The $\mathrm{M}_{\mathrm{w}}$ is calculated as the average of the molecular mass distribution considering the weight of each polymer fraction, whereas $M_{n}$ takes the number of molecules of each fraction into consideration. Therefore, smaller molecules have a greater impact on $\mathrm{M}_{\mathrm{n}}$ than on $\mathrm{M}_{\mathrm{w}}$. No significant differences in molecular mass were detected between the untreated, autoclaved, $\mathrm{scCO}_{2}$ treated and UV-irradiated $\mathrm{MC}$ for either $\mathrm{M}_{\mathrm{w}}$ or $\mathrm{M}_{\mathrm{n}}$. However, the $\gamma$-irradiated $\mathrm{MC}$ had a significantly reduced $\mathrm{M}_{\mathrm{w}}$ and $\mathrm{M}_{\mathrm{n}}$ (Fig.2, A-B). The measured polydispersity index used to indicate how far from a uniform distribution (PDI $=1$ ) a sample is, was seen to be significantly reduced for the $\gamma$-irradiated MC when compared to the nonsterilised and autoclaved samples, but not from UV and $\mathrm{scCO}_{2}$ treated $\mathrm{MC}$ (Fig.2, A-B).

\section{Alg/MC paste characterisation}

The sterilised MC variants were used to prepare plotting pastes by mixing the MC powder with a $3 \%$ alginate solution, the untreated MC formulation served as a control. The rheological and printing properties of the pastes were compared.

All pastes demonstrated shear thinning behaviour with a decrease in viscosity under shear strain (Fig.3, A). At a defined shear rate of $10 \mathrm{~s}^{-1}$ the viscosity measurements of the alg/MC pastes showed no significant difference between the non-sterilised ( $308 \pm 7.5 \mathrm{~Pa}$-s), autoclaved $(291 \pm 13.3 \mathrm{~Pa}-\mathrm{s})$ and $\mathrm{scCO}_{2}(273 \pm 27.3 \mathrm{~Pa}-\mathrm{s})$ group. The UV group had a significantly reduced viscosity of $224 \pm 12.4 \mathrm{~Pa}$-s followed by the $\gamma$-irradiation group with the strongly reduced viscosity of $11 \pm 1.5$ Pa-s (Fig.3, B).

All pastes were printable, however the increased flow rate and decreased viscosity of the $\gamma$-treated material required adaptation of the printing conditions (see section 2.5) and needed immediate crosslinking to achieve structural integrity and to preserve macropores (Fig.3, C). The effects of these parameter changes to print pressure and speed would cause a different shear stress applied to the plotted cell-laden material as well as alter the material volume extruded per scaffold. It was for this reason that $\gamma$-irradiation was not considered suitable for precise 3D bioplotting. Furthermore, the $\gamma$-sterilised scaffolds lacked form stability, especially on layers printed at increasing height, and so could not be appropriately used for mechanical testing. The use of $\gamma$-irradiation was not taken forward for cell inclusion, as variation from the other types was considered too high for a fair comparison.

\section{Alg/MC scaffold evaluation}

$\mathrm{MC}$ as a thickener agent has been previously employed as a temporary additive used to improve alginate printability for $3 \mathrm{D}$ bioplotting. The $\mathrm{MC}$ component is not ionically crosslinked by $\mathrm{Ca}^{2+}$ and has been shown to be released from the scaffolds over time [10]. In order to evaluate the impact of the MC sterilisation methods on that behaviour, the MC content of the scaffolds prepared from the different alg/MC pastes was visualised by chlorine-zinciodine staining. After 1 day of incubation under cell culture conditions all scaffold types appeared dark violet without any difference, indicating a high MC content (Fig.4, A, first column). After 7 days (Fig.4, A, second column) the intensity was visually unchanged for all MC types except for the $\gamma$-irradiation group; which had almost completely released MC from 
the scaffold. Dark staining remained within the UV, autoclaved and $\mathrm{scCO}_{2}$ scaffolds until the end of the study at day 21 (Fig.4, A, third column).

To quantitatively assess the effects of the different sterilisation treatments on MC release from the scaffolds over time, the concentration of released MC in collected supernatants was calculated cumulatively using Mykoval ${ }^{\mathrm{TM}}$ fluorescence intensity measurements (Fig.4, B). The untreated variant had consistently the lowest MC release with approximately $20 \%$ of the total MC content measured in the supernatant after crosslinking and 2 hours incubation (depicted as one cumulative data point at the $2 \mathrm{~h}$ mark) and only a marginal release after the 48 hours' time point. The $\gamma$-irradiated MC was released to the significantly highest extent for all time points tested: After 11 days, $100 \%$ of the $\gamma$-irradiated MC was released, with $80 \%$ already present in the supernatants after crosslinking and 2 hours incubation. Unlike the $\gamma$-treated samples, sterilisation by $\mathrm{scCO}_{2}$, autoclaving and UV-irradiation evoked a release of approximately $25 \%$ of total MC content after crosslinking and 2 hours incubation, followed by a moderate release until 48 hours and no (autoclave) or very low (UV and $\mathrm{scCO}_{2}$ ) additional release later on. At late time points (days 14, 19 and 21), the $\mathrm{scCO}_{2}$ scaffolds released significantly higher amounts compared to the autoclave and UV scaffold types (Fig.4, B).

The investigation of the scaffold surface microstructures using SEM imaging showed evenly distributed micropores for all MC variants (Fig.4, C, I). This indicates that the presence of MC itself, independent of size or release, was sufficient to cause micropores in the otherwise even surface of alginate (as was shown by Schütz et al [10]). However, a variation in pore structure was apparent on the alg/MC scaffolds prepared using the differently treated $\mathrm{MC}$, this effect is most evident on the 10,000 x magnification images (Fig.4, C, II). In scaffolds of the $\gamma$-irradiation group, symmetrical, rounded and regularly distributed pores were present, whereas in the other treatment types, in which a higher content of MC remained within the crosslinked alginate network, the pores were seen to be more fibrous and cavernous (Fig.4, C, II).

The compressive strength of the alg/MC scaffolds was the highest on day 1 of testing for all MC variants but decreased significantly between day 1 and 10 (Fig.5, A). On day 1 the hydrogel scaffold of the UV group had the significantly highest compressive strength of 45.2 $\pm 8.0 \mathrm{MPa}$. No significant difference could be determined between the autoclaved and $\mathrm{scCO}_{2}$ variants with values of $32.1 \pm 6.8$ and $27.7 \pm 4.6 \mathrm{MPa}$ respectively (Fig.5, A). Between days 10 and 21, a significant decline was observed for the $\mathrm{scCO}_{2}$ and $\mathrm{UV}$-treated $\mathrm{MC}$ variants. No significant differences were seen between the sterilised MC variants on day 10 or 21 of measurement.

Further indication of mechanical stability was the release of calcium ions during incubation. The amount of calcium released on days 1 and 10 (Fig.5, B) showed no significant differences between the different MC sterilisation groups. On day 21 the autoclaved variant had a significantly higher calcium release than the $\mathrm{scCO}_{2}$ variant, with values of $42.7 \pm 5.4$ $(\mu \mathrm{mol})$ and $28.8 \pm 3.9(\mu \mathrm{mol})$ respectively. The UV samples $(31.3 \pm 7 \mu \mathrm{mol})$ had no significant differences to either the autoclaved or $\mathrm{scCO}_{2}$ measurements. For all $\mathrm{MC}$ types, there was a significant increase in calcium release from day 1 to day 10. Differences in calcium release between day 10 and 21 were not significant for the $\mathrm{scCO}_{2} \mathrm{MC}$ type but showed a significant increase in release for the UV and autoclave treated scaffolds. 


\section{Cell compatibility of $\mathrm{Alg} / \mathrm{MC}$ scaffolds - towards cartilage tissue engineering}

For bioplotting of alg/MC scaffolds laden with bovine primary chondrocytes, autoclaved, $\mathrm{scCO}_{2}$ treated and UV-irradiated MC types were used and compared regarding their influence on cell compatibility.

The UV and the $\mathrm{scCO}_{2}$ variants showed consistent survival across all time intervals. However, in the autoclaved group, values for the percent of live cells on day $1(65.89 \% \pm 10.6)$ were significantly higher than on day $21(54.6 \pm 9.4)$ but with no significant differences between days 7, 14 and 21 (Fig.6, A). Values determined for the MC variants treated with UV and autoclave had no significant differences in cell survival on days 1, 7, 14 and 21. The MC variants treated with UV and autoclave supported a significantly higher cell survival within the alg/MC hydrogel scaffolds than compared to the $\mathrm{scCO}_{2}$-treated $\mathrm{MC}$ type on days 1,7 and 14 . By day 21 , only cell viability within UV scaffolds remained significantly higher than the $\mathrm{scCO}_{2}$ scaffold (Fig.6, A). On the whole, a higher number of viable cells was visible in the autoclave and the UV group in comparison to the scCO2 group (Fig.6, C).

The autoclaved MC type showed no significant change in DNA content over the whole cultivation time. DNA content of the $\mathrm{scCO}_{2}$ variant was significantly higher on day 1 and 7 compared to day 14 and 21. The DNA content of the UV variant showed a significant increase between day 1 and day 7, which then plateaued (Fig.6, B).

On day 1 there was no significant difference in the DNA content between the MC treatment types tested (Fig.6, B). On day 7 the scaffolds derived from UV-irradiated MC had the highest DNA content. On days 14 and 21, both the UV and the autoclaved variant had a significant higher DNA content than the $\mathrm{scCO}_{2}$ type.

In order to study extracellular matrix production, the histological staining of proteoglycans was performed with safranin-O on BPC-laden and BPC-free (control) scaffolds at regular intervals throughout culture duration. After 21 days in culture the $\mathrm{scCO}_{2}$ samples showed a visibly lower safranin-O stain intensity with sparse regions of proteoglycan when compared to the autoclaved and UV variants (Fig.7, A, I-III). Little or no safranin-O staining was present for day 7 or cell free samples for all MC treatment types tested confirming that the staining reveals matrix production by the cells (Fig.7, A, IV\&V).

The semi-quantitative analysis of the histology images indicated a significantly reduced stain coverage for the $\mathrm{scCO}_{2}$ samples in comparison to the $\mathrm{UV}$ and autoclaved samples, on days 7, 14 and 21 (Fig.7, B-C). Image assessment for the UV and autoclaved sample types revealed no significant differences with regard to stain intensity and coverage (Fig.7, B). On days 7 and 21 safranin-O stain intensity was higher in the UV and autoclave group than seen for the $\mathrm{scCO}_{2}$ samples; on day 14 no significant difference could be stated between the autoclaved and $\mathrm{scCO}_{2}$ variants (Fig.7, C). For the UV and autoclaved variants, a high area coverage was seen from day 7 on that did not increase overtime, however, the stain intensity did increase from day 7 to 21 . The $\mathrm{scCO}_{2}$ type showed a significant increase in intensity and coverage from day 7 to 14 but then showed no significant difference in either parameter by day 21 (Fig.7, B-C). 


\section{Discussion}

Biological scaffolds for medical purposes must be manufactured in a reproducible, controlled and sterile fashion [51]; and should be achieved using conditions possible for the inclusion of biological components such as living cells. The use of suitable sterilisation methods is therefore an essential step in the development of a clinically feasible regenerative approach. However, the identification of appropriate sterilisation methods is often underinvestigated and can hold numerous implications on material and cell behaviour; affecting both the feasibility for bioprinting and therapeutic validity. In the present study, we systematically investigated the influence of four different sterilisation methods on methyl cellulose, a relevant material for 3D printing application [17], used as a thickening additive to make cell-embedding hydrogels applicable for extrusion-based bioprinting [13-16, 52], on important characteristics of the alg/MC paste and crosslinked scaffolds.

\section{MC sterilisation effects on alg/MC paste properties for extrusion-based 3D printing}

For extrusion, the rheological properties of the plotting material are the essential parameters. In this study the viscosity measurements revealed that in contrast to autoclaving and $\mathrm{scCO}_{2}$ treatment, sterilisation by irradiation of the $\mathrm{MC}$ resulted in a significantly reduced viscosity of the alg/MC bioink (Fig.3, A-B). However, the type and dose of irradiation energy determined the strength of this effect: the non-ionizing lower energy photons of UV-irradiation resulted only in a slight reduction, whereas the high energy ionizing input from $\gamma$-irradiation led to a dramatic reduction of viscosity. The alg/MC paste derived from $\gamma$-irradiated $\mathrm{MC}$ showed a more fluid like behaviour which caused a loss of shape fidelity limiting the overall height and macropore structure upon printing. Although $\gamma$-irradiation has been commonly applied for the sterilisation of biomaterials for use in the manufacturing of biomedical products [53], its destructive action is known. Exposure to $\gamma$-irradiation has been demonstrated to degrade polysaccharides including starch, cellulose, and pectin by the cleavage of the glycosidic bonds [29,54]. The common result of this is a decreased viscosity and molecular mass [29]. Similarly, the strong decrease of viscosity of the alg/MC paste in the present study was accompanied by a marked reduction of the molecular mass of $\gamma$-treated MC (Fig.2, A-B).

In fact, the GPC measurements indicated that treatment of $\mathrm{MC}$ by autoclaving, $\mathrm{scCO}_{2}$ and UV-irradiation did not result in a significant shift of the molecular mass although slight changes can be assumed. With the PDI being slightly reduced after treatment via $\mathrm{scCO}_{2}$ and $\mathrm{UV}$ it could be argued that these methods led to cleavages in the larger molecules as well, but as this was not accompanied by a clear reduction in either $\mathrm{M}_{\mathrm{w}}$ or $\mathrm{M}_{\mathrm{n}}$ it is difficult to draw any definitive conclusions (Fig.2, A-B). The $\gamma$-irradiation on the other hand led to a marked decrease in both number and size of larger molecules; this led to a more even distribution of MC chain size, indicated by a much lower PDI. It should be noted that the non-sterilised MC showed high variation in $\mathrm{Mw}$, as the material was inherently inhomogeneous with chain lengths ranging from hundreds to tens of thousands of molecules. However, sterilisation by $\gamma$-irradiation resulted in an increased cleavage of the larger molecules and consequently in a decreased chain length variability. The loss of high molecular mass chains is most likely the cause for its much less viscous behaviour. As such, it can be surmised from this outcome that $\gamma$-sterilisation is not 
an appropriate method for MC treatment for the use of alg/MC extrusion due to its dramatically altered material properties.

In case of the alg/MC paste derived from UV-treated MC the slight reduction of viscosity did not affect the printability or structural integrity of the material. The viscosity measurements were taken after a swelling period of 18 hours to limit changes in swell between measurements affecting results. However, singular measurements taken after 2 hours (reflecting a similar swell time applied for the cell printing experiments) revealed viscosity values in a range of 50$70 \mathrm{~Pa}-\mathrm{s}$. When this is compared to the 200-300 Pa-s reached after overnight swelling it seems that longer swelling time maximised the different effects of the MC sterilisation on viscosity (Fig.3, B). Therefore, although the viscosity of the UV type was reduced following overnight swelling, this effect was not seen to change material behaviour during extrusion and deposition for the cell encapsulation experiments. Similar plotting parameters were applied for the pastes produced with autoclaved, $\mathrm{UV}, \mathrm{scCO}_{2}$ treated or non-sterilised $\mathrm{MC}$; they all produced defined and structurally stable scaffolds with little variation between strand diameters (Fig.3, C; Fig.4, A; for quantitative analysis see supplementary Fig.S4) or scaffold heights. In addition, throughout the 21-day investigation the structural integrity and shape fidelity of all scaffold types were maintained. It can therefore be stated that based on print quality alone the $\mathrm{scCO}_{2}$, autoclaving and UV are all suitable MC sterilisation methods, with an equal feasibility for successful extrusion of alg/MC.

\section{MC sterilisation effects on alg/MC scaffold characteristics}

In our previous work, we observed a successive release of $\mathrm{MC}$ from the hydrogel scaffolds after ionic crosslinking of the alginate chains [10]. Herein, we found that the release of MC from the scaffolds and finally their composition strongly depends on the method used for MC sterilisation. By applying chlorine-zinc-iodine staining, we confirmed the former observation for the $\gamma$-irradiated MC group, however, no apparent visual difference could be seen between the samples of the other sterilisation methods over time (Fig.4, A). This indicated that no further MC release occurred in samples when MC was sterilised by autoclaving, $\mathrm{UV}$ or $\mathrm{scCO}_{2}$ treatment. However, considering the oversaturated character of the chlorine-zinc-iodine staining we aimed to gain a more differentiated insight into MC release from the different variants and applied an assay based on Mykoval $^{\mathrm{TM}}$ fluorescence staining which was described here for the first time as a quantitative detection method for methyl cellulose concentration in solution. The findings verified the complete release of $\gamma$-irradiated MC and revealed a partial release of $\mathrm{MC}$ sterilised by autoclaving, $\mathrm{UV}$ and $\mathrm{scCO}_{2}$ treatment as well as of untreated $\mathrm{MC}$ (Fig.4, A-B).

It should be noted that our quantification approach might have been limited in the case of higher MC concentrations due to fluorescence extinction. However, in the range that was relevant for MC concentration in the supernatant (as presented here $<0.05 \%$ ) this effect was not apparent (not shown). All the calibration series were set up in culture medium, however, the early samples taken after crosslinking stages were in $100 \mathrm{mM} \mathrm{CaCl}_{2}$ solution. Therefore, a slight deviation of fluorescence intensity could have occurred due to effects of different ionic strength and/or optic properties (phenol red, proteins). Furthermore, an unspecific reaction of the fluorophore with dissolved alginate chains and molecules was detected, however, only for concentrations higher than the observed alginate release from the scaffolds after $\mathrm{Ca}^{2+}$ crosslinking. For analytical calculation by non-linear regression in our approach, the model of a saturated one-site-binding mechanism was chosen although the exact mechanism of the 
fluorophore reaction using this type of cellulose detection reagents is not described in detail $[37,55]$.

Nevertheless, to approach the release behaviour of differently sterilised MC in a quantitative way, this method presented an attractive new option. Thus, a correlation between the MC molecule size $\mathrm{M}_{\mathrm{w}}$ (Fig.2, A-B) and the overall MC release (Fig.4 B) was discovered, indicating a dependence of $\mathrm{MC}$ release on the presence of large molecules, being inversely proportional. For instance, the high bulk release of MC from the $\gamma$-type scaffolds was due to the increased mobility of the shorter MC chains and ultimately led to a nearly MC-free alginate scaffold. In line with this view is our assumption that large molecule cleavages are present in the $\mathrm{scCO}_{2}$ and UV-treated MC fractions, as deduced from the slightly reduced PDI (Fig.2, A$\mathrm{B}$ ), and that the $\mathrm{scCO}_{2}$ and $\mathrm{UV}$ group also showed slightly higher $\mathrm{MC}$ release rates than the untreated or the autoclaved groups.

The use of SEM provided an insight into the scaffold microstructure, and variances could be observed between the different types (Fig.4, C). The $\gamma$-type scaffolds appeared to have smaller and more symmetrical pores when compared to the other samples. This result could reflect the less disturbed nature of the alginate matrix of the $\gamma$-type scaffolds, caused by the shorter MC chains and the early MC release, which happened to a large extent already during incubation in the crosslinking solution. The more fibrous and cavernous pores observed in the autoclaved, $\mathrm{scCO}_{2}$ and UV-treated groups could have been induced by the presence of $\mathrm{MC}$ molecules with a higher $\mathrm{M}_{\mathrm{w}}$ breaking up the alginate network.

The calcium release for all scaffolds showed an increase with time which is expected of ionically crosslinked alginate scaffolds [56] and corresponds to the mechanical strength of the scaffolds decreasing over time. On days 1 and 10 no significant difference between the calcium release from the different scaffolds types were observed, whereas on day 21 the autoclaved type had the significantly highest release values and the UV-type the lowest (Fig.5, B). The effect of these differences however was not markedly observed in the physical attributes of the day 21 scaffolds (Fig.5, A). Currently, the interaction of alginate and methylcellulose is not fully elucidated, and the interaction potential between the particles should be further investigated to allow for a better insight, for example by determination of the second cross virial coefficient via osmometry [57]. Despite the decrease in strength overtime and internal degradation seen for all variants, caused by cumulative calcium release, the volumetric and external appearance of the scaffolds remained unchanged throughout for all MC types, demonstrated by the consistent strand width measurements (Fig.S4).

\section{MC sterilisation effects on cell survival and tissue formation in alg/MC scaffolds}

The live/dead staining results clearly demonstrated a significantly higher viability in alg/MC scaffolds prepared with autoclaved and UV-treated $\mathrm{MC}$, when compared to $\mathrm{scCO}_{2}$ sterilisation (Fig.6, A, C). Despite their very different methods of application, the UV and autoclaved variants showed no significant difference in cell survival on days 1, 7 or 21 of scaffold culture. The use of $\mathrm{scCO}_{2}$ for biomaterial sterilisation is a relatively new application not yet widely utilised. This method was reported to be an effective, gentle and also noncytotoxic low-temperature alternative for sterilisation of sensitive biomaterials [26,58]. However, although it is known that after $\mathrm{scCO}_{2}$ sterilisation the treated material should be left to ventilate before contact with cells, the timing of this has not been optimised. Hence, a 
possible reason for the reduced cell survival in the hydrogel matrix derived from $\mathrm{scCO}_{2}$ treated MC could be associated with the chemical additives used in the sterilisation process.

In post-experiment testing hydrogen peroxide was found to be present in $\mathrm{scCO}_{2}$ treated MC to notable amounts for up to 7 days after sterilisation, but after 14 days of letting the sachet of MC air out at room temperature only trace amounts remained, and no residue was seen after 21 days of storage (see supplementary Fig.S5). No evidence of peracetic acid could be detected even at the earliest time points analysed (data not shown). The untreated MC tested showed no traces of either peracetic acid or hydrogen peroxide. A minimum of just 10 days before use was employed within this study. Thus, it is possible that trace amounts of hydrogen peroxide could have contributed to the reduced cell survival within the $\mathrm{scCO}_{2}$ derived scaffolds. This result highlights that the use of $\mathrm{scCO}_{2}$ as a sterilisation method for biomaterials requires further optimisation and careful verification before it can be seen as a valid option for clinical applications. Furthermore, with this work it was possible to show that both UV-irradiation and autoclaving, despite being known as harsh methods where biopolymers are concerned [22], did not have a negative influence on $\mathrm{MC}$, but are much more widely established than $\mathrm{scCO}_{2}$.

The DNA content results coincided with the cell survival data (Fig.6, B). From day 7 the UV and autoclaved MC scaffold types had a higher DNA content than the $\mathrm{scCO}_{2}$ group. And whereas a constant or even increasing DNA content was shown for the autoclaved and UVtreated MC type scaffolds, the DNA content of the $\mathrm{scCO}_{2}$ samples decreased over time. These results demonstrated again the positive effect of the UV and autoclaved MC treatments on cell response in contrast to those of the $\mathrm{scCO}_{2}$ treatment. In addition, the findings of the histology staining further corroborate this effect (Fig.7, A-C). A marked visual difference in stain intensity was observed between the $\mathrm{scCO}_{2}$ samples compared to the UV and autoclaved, for days 7, 14 and 21. For each time point the $\mathrm{scCO}_{2}$ samples gave a sparse and less intense staining for proteoglycans than the scaffolds derived from the autoclaved and UV-treated MC indicating that the higher percent of living and metabolically active cells present on days 1, 7 and 14 consequently laid down a denser extracellular matrix by day 21 .

\section{Conclusion}

UV-irradiation, autoclave and $\mathrm{scCO}_{2}$ treatment applied for $\mathrm{MC}$ powder sterilisation were highly comparable with regards to printability of the resulting alg/MC pastes - defined strands and structurally stable scaffolds could be produced which maintained their shape over the 21day testing period. The use of $\gamma$-irradiated MC powder resulted in an alg/MC paste with a substantial reduction in viscosity, leading to poorly formed scaffolds. A marked decrease in molecular mass of the $\gamma$-irradiated MC was attributed to its very different material behaviour, that in addition led to a complete release of MC over time. Only slight variations between scaffold properties were observed for the other tested sterilisation methods with molecular masses closer in range, which indicates that autoclaving was not more intense than the low temperature $\mathrm{scCO}_{2}$ treatment. Investigations of cell compatibility and tissue formation revealed the UV-irradiated and autoclaved MC to be the best candidates for alg/MC cell-laden printing. However, as the UV-treatment is not considered a fully effective method for the terminal stage sterilisation of all biological life, we propose autoclaving for sterilisation of powdered MC which is a widely accessible and clinically approved method. The application of $\mathrm{scCO}_{2}$ 
treatment for sterilisation of sensitive biopolymers requires further optimisation before it can be considered a viable sterilisation approach for bioprinting purposes. MC with alginate was demonstrated to be highly compatible with chondrocytes, with wider implications as a promising material for cartilage regeneration approaches using bioplotting.

In summation, this investigation demonstrates that the sterilisation method used can have a direct impact on the physical properties of the material as well as on the behaviour of embedded cells. Although the exact mechanism of this interaction could not be fully elucidated, our results suggest that the difference between the MC sterilisation types was not due to a chemical change, as shown by consistent FT-IR spectra, but must be the result of a physical alteration to matrix distributions caused by the observed differences in molecular mass. The outcome of this investigation highlights the necessity for appropriate sterilisation method selection for biomaterial compatibility and feasibility for 3D bioplotting, with a particular relevance for recently explored cellulose based bioinks.

\section{Acknowledgements}

The authors thank Ms Ortrud Zieschang for preparation of SEM samples, the microscopy facility CFCI of the TU Dresden for providing equipment and support in cell imaging as well as the Institute of Natural Materials Technology, Chair of Food Engineering at TU Dresden for the opportunity to perform gel permeation chromatography (GPC). This work was supported by the European Social Fund (ESF) and Free State of Saxony (Young Researchers Group IndivImp at Technische Universität Dresden), the German Research Society (DFG) as part of the priority program SPP 1934 as well as the German Center for Diabetes Research (DZD). Partial funding was received from a Santander Research Scholarship, awarded to Ella Hodder.

\section{Supplementary figures}

\section{References}

1. Liaw C-Y, Guvendiren M. Current and emerging applications of 3D printing in medicine. Biofabrication. 2017; 9: (2) 24102.

2. Ventola CL. Medical applications for 3D printing: current and projected uses. P\&T. 2014; 39: (10) 704-711.

3. Billiet T, Vandenhaute M, Schelfhout J, Vlierberghe S Van, Dubruel P. A review of trends and limitations in hydrogel-rapid prototyping for tissue engineering. Biomaterials. 2012; 33: (26) 6020-6041.

4. Malda J, Visser J, Melchels FP, Jüngst T, Hennink WE, Dhert WJA, Groll J, Hutmacher DW. 25th anniversary article: Engineering hydrogels for biofabrication. Adv Mater. 2013; 25: (36) 5011-5028. 
5. Lee KY, Mooney DJ. Alginate: Properties and biomedical applications. Prog Polym Sci. 2012; 37: (1) 106-126.

6. Lee KY, Rowley JA, Eiselt P, Moy EM, Bouhadir KH, Mooney DJ. Controlling mechanical and swelling properties of alginate hydrogels independently by crosslinker type and cross-linking density. Macromolecules. 2000; 33: (11) 4291-4294.

7. Joydip K, Jin-Hyung S, Jinah, Jang S, Sung WK, Dong-Woo C. An additive manufacturing-based PCL-alginate- chondrocyte bioprinted scaffold for cartilage tissue engineering. J Tissue Eng Regen Med. 2013; 9: (11) 1286-1297.

8. Fedorovich NE, Schuurman W, Wijnberg HM, Prins H-J, van Weeren PR, Malda J, Alblas J, Wouter JA, Dhert MD. Biofabrication of osteochondral tissue equivalents by printing topologically defined, cell-laden hydrogel scaffolds. Tissue Eng Part C. 2012; 18: (1) 33-44.

9. Maher PS, Keatch RP, Donnelly K, Mackay RE, Paxton JZ. Construction of 3D biological matrices using rapid prototyping technology. Rapid Prototyp J. 2009; 15: (3) 204-210.

10. Schütz K, Placht A-M, Paul B, Brüggemeier S, Gelinsky M, Lode A. Threedimensional plotting of a cell-laden alginate/ methylcellulose blend: towards biofabrication of tissue engineering constructs with clinically relevant dimensions. $\mathbf{J}$ Tissue Eng Regen Med. 2017; 11: (5) 1574-1587.

11. Zehnder T, Sarker B, Boccaccini AR, Detsch R. Evaluation of an alginate-gelatine crosslinked hydrogel for bioplotting. Biofabrication. 2015; 7: (2) 25001.

12. Hwang CM, Ay B, Kaplan DL, Rubin JP, Marra KG, Atala A, Yoo JJ and Lee SJ. Assessments of injectable alginate particle-embedded fibrin hydrogels for soft tissue reconstruction. Biomed Mater. 2013; 8: (1) 14105.

13. Lode A, Krujatz F, Brüggemeier S, Quade M, Schütz K, Knaack S, Weber J, Bley T, Gelinsky M. Green bioprinting: Fabrication of photosynthetic algae-laden hydrogel scaffolds for biotechnological and medical applications. Eng Life Sci. 2015; 15: (2) 177-183.

14. Seidel J, Ahlfeld T, Adolph M, Kümmritz S, Steingroewer J, Krujatz F, Bley T, Gelinsky M, Lode A. Green bioprinting: extrusion-based fabrication of plant cellladen biopolymer hydrogel scaffolds. Biofabrication. 2017; 9: (4) 45011.

15. Li H, Tan YJ, Leong KF, Li L. 3D Bioprinting of highly thixotropic alginate/methyl cellulose hydrogel with strong interface bonding. ACS Appl Mater Interfaces. 2017; 9: (23) 20086-20097.

16. Ahlfeld T, Cidonio G, Kilian D, Duin S, Akkineni A R, Dawson J I, Yang S, Lode A, Oreffo R O C, Gelinsky M. Development of a clay based bioink for 3D cell printing for skeletal application. Biofabrication. 2017; 9: (3) e034103 (16 pages).

17. Wang Q, Sun J, Yao Q, Ji C, Liu J, Zhu Q. 3D printing with cellulose materials. Cellulose. 2018; 1-27.

18. Nasatto PL, Pignon F, Silveira JLM, Duarte MER, Noseda MD, Rinaudo M. Methylcellulose, a cellulose derivative with original physical properties and extended applications. Polymers. 2015; 7: (5) 777-803.

19. Sarma NJ, Takeda A, Yaseen NR. Colony forming cell (CFC) assay for human hematopoietic cells. J Vis Exp. 2010; 46: 3-7. 
20. Munarin F, Bozzini S, Visai L, Tanzi MC, Petrini P. Sterilization treatments on polysaccharides: Effects and side effects on pectin. Food Hydrocoll. 2013; 31: (1) 74-84.

21. Mendes GCC, Brandão TRS, Silva CLM. Ethylene oxide sterilization of medical devices: A review. Am J Infect Control. 2007; 35: (9) 574-81.

22. Dai Z, Ronholm J, Tian Y, Sethi B, Cao X. Sterilization techniques for biodegradable scaffolds in tissue engineering applications. J Tissue Eng. 2016; 7: 1-13.

23. Ahmed EM. Hydrogel: Preparation, characterization, and applications: A review. J Adv Res. 2015; 6: (2)105-121.

24. Karajanagi SS, Yoganathan R, Mammucari R, Park H, Cox J, Zeitels SM, Langer R, Foster NR, Sandeep S. Application of a dense gas technique for sterilizing soft biomaterials. Biotechnol Bioeng. 2011; 108: (7) 1716-1725.

25. Damar S, Balaban MO. Review of Dense Phase $\mathrm{CO}_{2}$ Technology: Microbial and enzyme inactivation. Journal of Food Science. 2006; 71: (1) 1-11.

26. Bernhardt A, Wehrl M, Paul B, Hochmuth T, Schumacher M, Schütz K, Gelinsky M. Improved sterilization of sensitive biomaterials with supercritical carbon dioxide at low temperature. PLoS One. 2015; 10: (6) e0129205 (19 pages).

27. Spilimbergo S, Dehghani F, Bertucco A, Foster NR. Inactivation of bacteria and spores by pulse electric field and high-pressure $\mathrm{CO}_{2}$ at low temperature. Biotechnol Bioeng. 2003; 82: (1) 118-125.

28. Collier JP, Sperling DK, Currier JH, Sutula LC, Saum KA, Mayor MB. Impact of gamma sterilization on clinical performance of polyethylene in the knee. $\mathrm{J}$ Arthroplasty. 1996; 11: (4) 377-389.

29. El-ashhab F, Sheha L, Abdalkhalek M, Khalaf HA. The influence of gamma irradiation on the intrinsic properties of cellulose acetate polymers. J Assoc Arab Univ Basic Appl Sci. 2013; 14: (1) 46-50.

30. Palmer I, Clarke S, Nelson J, Schatton W, Dunne N, Buchanan F. Identification of a suitable sterilisation method for collagen derived from a marine Demosponge. International Journal of Nano and Biomaterials. 2012; 4: (2) 148-163.

31. Stoppel WL, White JC, Horava SD, Henry AC, Roberts SC, Bhatia SR. Terminal sterilization of alginate hydrogels: Efficacy and impact on mechanical properties. $\mathrm{J}$ Biomed Mater Res - Part B Appl Biomater. 2014; 102: (4) 877-884.

32. Goldman M, Gronsky R, Ranganathan R, Pruitt L. The effects of gamma radiation sterilization and ageing on the structure and morphology of medical grade ultra high molecular weight polyethylene. Polymer (Guildf). 1996; 37: (14) 2909-2913.

33. Maslennikova A, Kochueva M, Ignatieva N, Vitkin A, Zakharkina O, Kamensky V, Sergeeva E, Kiseleva E, Bagratashvili V. Effects of gamma irradiation on collagen damage and remodeling. Int J Radiat Biol. 2015; 91: (3) 240-247.

34. Meechan P, Wilson C. Use of ultraviolet lights in biological safety cabinets: A contrarian view. Appl Biosaf. 2006; 11: (4) 222-227.

35. Huebsch N, Gilbert M, Healy KE. Analysis of sterilization protocols for peptidemodified hydrogels. J Biomed Mater Res - Part B Appl Biomater. 2005; 74: (1) 440447.

36. Wilda $\mathrm{H}$, Gough JE. In vitro studies of annulus fibrosus disc cell attachment, differentiation and matrix production on PDLLA/45S5 Bioglass composite films. Biomaterials. 2006; 27: (30) 5220-5229. 
37. T Reza A, B Nicoll S. Hydrostatic pressure differentially regulates outer and inner annulus fibrosus cell matrix production in 3D scaffolds. Annals of Biomedical Engineering. 2008; 36: (2) 204-213.

38. Bryant SJ, Anseth KS. Hydrogel properties influence ECM production by chondrocytes photoencapsulated in poly (ethylene glycol ) hydrogels. J Biomed Mater Res. 2002; 59: (1) 63-72.

39. Pan T, Song W, Cao X, Wang Y. 3D Bioplotting of gelatin/alginate scaffolds for tissue engineering: influence of crosslinking degree and pore architecture on physicochemical properties. J Mater Sci Technol. 2016; 32: (9) 889-900.

40. Leo WJ, Mcloughlin AJ, Malone DM. Effects of Sterilization Treatments on Some Properties of Alginate Solutions and Gels. Biotechnol. 1990; 6: (1) 51-53.

41. Stoppel WL, White JC, Horava SD, Henry AC, Roberts SC, Bhatia SR. Terminal sterilization of alginate hydrogels: Efficacy and impact on mechanical properties. J. Biomed. Mater. Res. - Part B Appl. Biomater. 2014; 102: (4) 877-884.

42. Young J, So J, Park H, Won J. Effects of intermittent hydrostatic pressure magnitude on the chondrogenesis of MSCs without biochemical agents under 3D co-culture. J Mater Sci: Mater Med. 2012; 23: (11) 2773-2781.

43. Park JY, Choi YJ, Shim JH, Park JH, Cho DW. J. Development of a 3D cell printed structure as an alternative to autologs cartilage for auricular reconstruction. Biomed. Mater. Res. - Part B Appl. Biomater. 2016; 105: (5) 1-13.

44. Kundu J, Shim JH, Jang J, Kim SW, Cho DW. An additive manufacturing-based PCL-alginate- chondrocyte bioprinted scaffold for cartilage tissue engineering. Tissue Eng. Regen. Med. 2013; 9: (11) 1286-1297.

45. Hall AC, Starks I, Shoults CL, Rashidbigi S. Pathways for K+ transport across the bovine articular chondrocyte membrane and their sensitivity to cell volume. Am J Physiol. 1996; 270: (5) C1300-C1310.

46. Fischbach C, Tessmar J, Lucke A, Schnell E, Schmeer G, Blunk T, GÖpferich A. Does UV irradiation affect polymer properties relevant to tissue engineering? Surf Sci. 2001; 491: (3) 333-345.

47. Janorkar A, Metters A, Hirt D. Degradation of Poly(L-Lactide) films under ultraviolet- induced photografting and sterilization conditions. Surface Science. 2006; 106: (2) 1042-1047.

48. Yixiang D, Yong T, Liao S, Chan CK, Ramakrishna S. Degradation of electrospun nanofiber scaffold by short wave length ultraviolet radiation treatment and its potential applications in tissue engineering. Tissue Eng Part A. 2008; 14: (8) 13211329.

49. Koch HH, Pimsler M. Evaluation of Uvitex 2B: a nonspecific fluorescent stain for detecting and identifying fungi and algae in tissue. Lab Med.1987; 18: (9) 603-606.

50. Rasconi S, Jobard M, Jouve L, Sime-Ngando T. Appl. Environ. Microbiol. 2009; 75 : (8) 2545-2553.

51. O’Brien F J. Biomaterials \& Scaffolds for Tissue Engineering. Materials Today. 2011; 14: (3) 88-95.

52. Law N, Doney, Glover H, Qin Y, Aman ZM, Sercombe TB, Liew LJ, Dilley RJ, Doyle BJ. J. Mech. Behav. Biomed. Mater. 2018; 77: 389-399.

53. Hugo WB. A brief history of heat, chemical and radiation preservation and disinfection. Int Biodeterior Biodegrad. 1995; 36: (3-4) 197-217. 
54. Hermannova M, Bezáková Z, Ebringerova A, Malovı A, Dřímalová E, Malovíková A, Ebringerová A, Velebný V. Effect of microwave irradiation on the molecular and structural properties of hyaluronan. Carbohydrate Polymers. 2008; 73: 640-646.

55. Hoffman AS. Hydrogels for biomedical applications. Advanced Drug Delivery Reviews. 2002; 54: 3-12.

56. Shahriari D, Koffler J, Lynam DA, Tuszynski MH, Sakamoto JS. Characterizing the degradation of alginate hydrogel for use in multilumen scaffolds for spinal cord repair. J Biomed Mater Res Part A. 2016; 104: (3) 611-619.

57. Ersch C, Linden E, Martin A, Venema P. Food Hydrocoll. 2016; 52: 991-1002.

58. Meyer M, Prade I, Leppchen-Fröhlich K, Felix A, Herdegen V, Haseneder R, Repkeb J-U. Sterilisation of collagen materials using hydrogen peroxide doted supercritical carbon dioxide and its effects on the materials properties. J Supercrit Fluids. 2015;

102: 32-39.

\section{Figure captions}

Fig. 1 FT-IR analysis of MC powder treated by different sterilisation methods (A) Example FT-IR spectrum of MC powder with labelled peaks, the spectra of all MC types measured (autoclaved, $\mathrm{scCO}_{2}$ treated, $\mathrm{UV}$ and $\gamma$-irradiated) were identical in appearance, (B) Identification of MC spectrum peaks.

Fig. 2 Gel permeation chromatography, weight and numerical average molecular mass $\left(\mathrm{M}_{\mathrm{w}}\right.$ and $\mathrm{M}_{\mathrm{n}}$ ) as well as PDI of MC powders prepared using non-sterilised MC and MC sterilised by autoclave, $\mathrm{scCO}_{2}$, UV and $\gamma$-irradiation. Mean $\pm \mathrm{stdev}, \mathrm{n}=3,{ }^{*} \mathrm{p}<0.05$ compared to the nonsterilised MC.

Fig.3 Viscosity measurements of alg/MC pastes.MC was mixed into dissolved alginate and left to swell overnight (A) Viscosity over shear rate of alg/MC pastes prepared using non-sterilised $\mathrm{MC}$ and $\mathrm{MC}$ sterilised by autoclave, $\mathrm{scCO}_{2}, \mathrm{UV}$ and $\gamma$-irradiation. One representative measurement is shown from each variant to demonstrate material behaviour. At all time points, the viscosity of the alg/MC paste with $\gamma$-irradiated $\mathrm{MC}$ was significantly lower than the other variants. (B) Viscosity of alg/MC pastes at a defined shear rate of $10 \mathrm{~s}^{-1}$ prepared with nonsterilised MC and sterilised MC. Mean \pm stdev, $n=3,{ }^{*} p<0.05$ compared to the non-sterilised MC. (C) Examples of plotted scaffolds prepared using non-sterilised MC and MC sterilised by autoclave, $\mathrm{scCO}_{2}$, UV and $\gamma$-irradiation (scale bars $=2 \mathrm{~mm}$ ).

Fig.4 Methyl cellulose content and microstructure of alg/MC scaffolds (A) Stereomicroscopy images of alg/MC scaffolds after 1, 7 and 21 days of cell culture stained with chlorine-zinciodine solution, prepared with untreated $\mathrm{MC}$ and sterilised $\mathrm{MC}$ via autoclave, $\mathrm{scCO}_{2}, \mathrm{UV}$ and $\gamma$-irradiation, (scale bars $=2 \mathrm{~mm}$ ). (B) Quantitative measurement of cumulative percentage of $\mathrm{MC}$ release over time. Mean $\pm \mathrm{stdv}, \mathrm{n}=3$. At all time points, release of the $\gamma$-irradiated $\mathrm{MC}$ was significantly higher; at days 14,19 and 21, release of the $\mathrm{scCO}_{2}$-treated $\mathrm{MC}$ was significantly higher than those of the autoclaved and UV-treated MC; after 21 days, release of all sterilised MC types was significantly higher than those of the untreated MC $\left({ }^{*} p<0.05\right)$. (C) Representative scanning electron microscopy images of alg/MC scaffolds prepared with the different MC variants at a magnification of x250 (I) and x10,000 (II) after 1 day of incubation under cell culture conditions (scale bars for $\mathrm{I}=200 \mu \mathrm{m}$ and $\mathrm{II}=50 \mu \mathrm{m}$ ). 
Fig.5 The effect of different sterilisation procedures on scaffold stability of cell-free scaffolds kept under cell culture conditions (A) Compressive strength of alg/MC scaffolds prepared with the $\mathrm{MC}$ variants treated with autoclave, $\mathrm{scCO}_{2}$ and $\mathrm{UV}$, on days 1,10 and 21 of incubation. Mean $\pm \operatorname{stdv}, n=12,{ }^{*} p<0.05$. (B) Cumulative calcium release by alg/MC scaffolds prepared using the different $\mathrm{MC}$ sterilisation methods, autoclaving, $\mathrm{scCO}_{2}$ and $\mathrm{UV}$, measured after 1, 10 and 21 days of incubation. Mean $\pm \operatorname{stdv}, n=9,{ }^{*} p<0.05$.

Fig.6 Cytocompatibility of BPC-laden alg/MC scaffolds prepared with MC treated by autoclave, $\mathrm{scCO}_{2}$ and UV at days 1, 7, 14 and 21 of cell culture (A) Survival of live BPC within the scaffolds, mean \pm stdv, $n=12,{ }^{*}<<0.05$. (B) Total DNA content of BPC embedded in the scaffolds, mean \pm stdv, $n=6, * p<0.05$. (C) Representative fluorescence micrographs of live/dead stained samples; live cells are shown in green and dead cells in red.

Fig.7 Glycosaminoglycan histology staining of BPC-laden and cell-free alg/MC scaffolds prepared with autoclave, $\mathrm{scCO}_{2}$ and UV treated $\mathrm{MC}$ (A) Histological images of scaffold sections, fixed at day 21 of cell culture and stained with safranin-O, taken by light microscopy (I) 20x magnification showing areas of high stain intensity (II) 20x magnification images to represent regions of low staining (III) 10x magnification images (IV) 10x magnification of a cell free scaffold prepared with autoclaved MC and fixed at day 14 of incubation under cell culture conditions(V) 20x magnification of BPC-laden scaffold prepared with autoclaved MC and fixed at day 7 of culture scalebars for all images represent $10 \mu \mathrm{m}$. (B) Percentage of image area covered by safranin-O staining in histology images (20x magnification) taken from scaffolds on days 7, 14 and 21 of cell culture. Mean $\pm s t d v, n=9$, ${ }^{*} p<0.05$. (C) Relative intensity of safranin-O staining taken from histology images (20x magnification) of scaffolds on days 7 , 14 and 21 of cell culture. Mean $\pm \operatorname{stdv}, n=9, * p<0.05$.

Supplementary Figure S1: FT-IR spectra of a 5\% alginate solution (A) before autoclaving and (B) after autoclaving. Samples were measured four times each, and the average major peak values were recorded. No significant differences between major peak values from the autoclaved and non-autoclaved alginate samples were detected and the spectra showed no apparent visible changes.

Supplementary Figure S2: Calibrated MC concentration curves via detected Mykoval ${ }^{\mathrm{TM}}$ fluorescence signal intensity, performed for each MC treatment type, excitation $400 \mathrm{~nm}$ and emission $450 \mathrm{~nm}$

Supplementary Figure S3: Normalised FT-IR spectra of MC powders non-sterilised and sterilised using the different methods.

Supplementary Figure S4: Mean width of alg/MC strands ( \pm stdv) produced by 3D bioplotting of alg/MC pastes prepared with sterilised $\mathrm{MC}$ via autoclave, $\mathrm{scCO}_{2}$ or UVirradiation after 1, 7, 14 and 21 days of incubation. Mean $\pm \operatorname{stdv}, n=3$.

Supplementary Figure S5: Hydrogen peroxide test strip results using non-sterilised and $\mathrm{scCO}_{2}$ treated $\mathrm{MC}$ dissolved in $\mathrm{ddH}_{2} \mathrm{O}$ tested 1, 7, 14 and 21 days after sterilisation. 